\title{
WHEN IS A LINEAR FUNCTIONAL MULTIPLICATIVE?
}

\author{
BY
}

\author{
M. ROITMAN AND Y. STERNFELD
}

\begin{abstract}
We prove here by elementary arguments a generalization of a theorem by Gleason, Kahane and Zelazko: If $\varphi$ is a linear functional on an algebra with unit $A$ such that $\varphi(1)=1$ and $\varphi(u) \neq 0$ for any invertible $u$ in $A$, then $\varphi$ is multiplicative, provided the spectrum of each element in $A$ is bounded. We present also other conditions which may replace the assumptions on $A$ in the theorem above.
\end{abstract}

1. Introduction. In [3], [4], and [7], Gleason, Kahane and Żelazko proved that in a Banach algebra with unit over the complex field $\mathbf{C}$, a linear functional which assigns the value 1 to the unit and which does not vanish on invertible elements, is multiplicative. Their proofs are analytic in nature.

In this article we generalize this result. By using elementary methods we show that the same result holds in any algebra with unit $A$ over $\mathbf{C}$ provided the spectrum of each element of $A$ is not too large. In particular, if for each $x \in A, \sigma(x)$ is not dense in $\mathbf{C}$ or $|\sigma(x)|<2^{\aleph_{0}}$, then the theorem holds (| $\mid$ stands here for cardinality). Throughout this paper $A$ will denote an algebra with unit over $\mathbf{C}$, so $\mathbf{C} \subseteq A$. We denote the group of invertible elements of $A$ by $A^{*}$. The resolvent of an element $x \in A$ is the set $\rho(x)=\left\{\lambda \in \mathbf{C}: x-\lambda \in A^{*}\right\}$ and its spectrum is $\sigma(x)=\mathbf{C} \backslash \rho(x)$. A linear functional $\varphi: A \rightarrow \mathbf{C}$ is called quasimultiplicative (q.m. in short) if $\varphi(1)=1$ and $0 \notin \varphi\left(A^{*}\right)$ (equivalently $\varphi(x) \in \sigma(x)$ for all $x \in A$ ). Obviously any nonzero multiplicative functional is q.m. $A$ is called ordinary if every q.m. functional on $A$ is multiplicative.

For a subset $S$ of $\mathbf{C}$ and scalars $a, b$, we use notations as

$$
\begin{aligned}
(S-a) /(S-b) & =\{(z-a) /(z-b): z \in S\} \quad(\text { provided } b \notin S), \\
S^{*} & =\{z \in S: z \neq 0\} \quad\left(\text { that is, } S^{*}=\mathbf{C}^{*} \cap S\right), \\
S^{2} & =\left\{z^{2}: z \in S\right\}, \quad S \cdot S=\left\{z_{1} z_{2}: z_{1}, z_{2} \in S\right\}, \quad \text { etc. }
\end{aligned}
$$

In $\$ 2$ we recall some known facts and prove a simple lemma from which the Gleason-Kahane-Żelazko theorem follows at once. In $\$ 3$ we study the local case: Given an element $x$ of an algebra $A$, consider the collection of all subalgebras with unit $B$ of $A$ which contain $x$ and such that $B^{*}=B \cap A^{*}$ (that is, for $y \in B$, $\left.\sigma_{B}(y)=\sigma_{A}(y)\right)$. Clearly the intersection $A(x)$ of all such subalgebras is still in this collection. It turns out that the ordinariness of $A(x)$ is determined by $\sigma(x)$ and we give some necessary and some sufficient conditions on $\sigma(x)$ for the ordinariness of

Received by the editors August 26, 1980.

AMS (MOS) subject classifications (1970). Primary 46H99.

() 1981 American Mathematical Society $0002-9947 / 81 / 0000-0407 / \$ 04.50$ 
$A(x)$. We also present examples which refute some possible conjectures in this connection. In $\$ 5$ we globalize the discussion and deal with the ordinariness of general algebras. In $\$ 4$ we study q.m. functionals on the algebra of all entire functions.

2. A generalization of the Gleason-Kahane-Żelazko theorem. The following lemma, which is a part of the proof of Theorem 10.9 in [6, p. 233], will be proved here for the sake of completeness.

LEMMA 1. The following four conditions are equivalent for a linear functional $\varphi$ on an algebra $A$ with $\varphi(1)=1$.

(i) $\varphi(x)=0$ implies $\varphi\left(x^{2}\right)=0$ for $x \in A$.

(ii) $\varphi\left(x^{2}\right)=(\varphi(x))^{2}, x \in A$.

(iii) $\varphi(x)=0$ implies $\varphi(x y)=0$ for $x, y$ in $A$.

(iv) $\varphi(x y)=\varphi(x) \varphi(y), x, y$ in $A$.

Proof. (i) $\Rightarrow$ (ii) $\varphi(1)=1$ implies $\varphi(x-\varphi(x))=0$. By (i)

$$
0=\varphi\left((x-\varphi(x))^{2}\right)=\varphi\left(x^{2}-2 x \varphi(x)+\varphi(x)^{2}\right)=\varphi\left(x^{2}\right)-\varphi(x)^{2},
$$

i.e., $\varphi\left(x^{2}\right)=\varphi(x)^{2}$.

(ii) $\Rightarrow$ (iii) By substituting $u+v$ for $x$ in (ii) we obtain

$$
\varphi(u v+v u)=2 \varphi(u) \varphi(v), \quad u, v \in A .
$$

Let $x, y$ be in $A$ with $\varphi(x)=0$. By (1) we obtain

$$
\varphi(x y+y x)=0 \text {. }
$$

Hence, by (ii), $\varphi\left((x y+y x)^{2}\right)=0$ also. Thus, from the identity $(x y-y x)^{2}=$ $2(x(y x y)+(y x y) x)-(x y+y x)^{2}$ it follows (using (1)) that

$$
\varphi\left((x y-y x)^{2}\right)=2 \varphi(x(y x y)+(y x y) x)=4 \varphi(x) \varphi(x y x)=0,
$$

and by (ii)

$$
\varphi(x y-y x)=0 .
$$

Adding (2) and (3) we obtain $\varphi(x y)=0$.

(iii) $\Rightarrow$ (iv) Let $x, y \in A . \varphi(x-\varphi(x))=0$; hence, by (iii),

$$
0=\varphi((x-\varphi(x)) y)=\varphi(x y-\varphi(x) y)=\varphi(x y)-\varphi(x) \varphi(y)
$$

and (iv) follows.

(iv) $\Rightarrow$ (i) is trivial.

LEMMA 2. Let $\varphi$ be a q.m. functional on an algebra $A$ and $x \in A$. If $\varphi(x)=0$ and $\varphi\left(x^{2}\right) \neq 0$, then $\sigma(x)$ is unbounded.

Proof. Consider the polynomial $P(\lambda)=\varphi\left((\lambda-x)^{n}\right)(n>2)$.

Let $\left\{\lambda_{i}\right\}_{i=1}^{n}$ be the roots of $P$. Then for each $1 \leqslant i \leqslant n, 0=P\left(\lambda_{i}\right)=\varphi\left(\left(\lambda_{i}-x\right)^{n}\right)$. Since $\varphi$ is q.m., $\left(\lambda_{i}-x\right)^{n} \notin A^{*}$, hence $\lambda_{i}-x \notin A^{*}$ also, i.e., $\left\{\lambda_{i}\right\}_{i=1}^{n} \subseteq \sigma(x)$. On the other hand,

$$
P(\lambda)=\lambda^{n}-n \varphi(x) \lambda^{n-1}+\left(\begin{array}{l}
n \\
2
\end{array}\right) \varphi\left(x^{2}\right) \lambda^{n-2}+\cdots=\prod_{i=1}^{n}\left(\lambda-\lambda_{i}\right) .
$$


Hence

$$
\sum_{i=1}^{n} \lambda_{i}=n \varphi(x)=0 \quad \text { and } \quad \sum_{i<j} \lambda_{i} \lambda_{j}=\left(\begin{array}{l}
n \\
2
\end{array}\right) \varphi\left(x^{2}\right)
$$

Thus

$$
0=\left(\sum_{i=1}^{n} \lambda_{i}\right)^{2}=\sum_{i=1}^{n} \lambda_{i}^{2}+2 \sum_{i<j} \lambda_{i} \lambda_{j}=\sum_{i=1}^{n} \lambda_{i}^{2}+n(n-1) \varphi\left(x^{2}\right)
$$

and

$$
n(n-1)\left|\varphi\left(x^{2}\right)\right|=\left|\sum_{i=1}^{n} \lambda_{i}^{2}\right|<\sum_{i=1}^{n}\left|\lambda_{i}\right|^{2}
$$

It follows that for some $1 \leqslant i \leqslant n,\left|\lambda_{i}\right|^{2} \geqslant(n-1)\left|\varphi\left(x^{2}\right)\right|$, and, since $n$ is arbitrary, $\sigma(x)$ is unbounded.

The Gleason-Kahane-Żelazko theorem is a particular case of the following

COROLlary. If for each $x \in A, \sigma(x)$ is bounded, then $A$ is ordinary.

Proof. Use Lemmas 1 and 2.

3. The local case. We denote by $\mathrm{C} X X]$ the ring of polynomials in the indeterminate $X$ and by $\mathbf{C}(X)$ the ring of rational functions of $X$.

Let $\sigma$ be a subset of $\mathrm{C}$. We denote by $A(\sigma)$ the following subalgebra of $\mathrm{C}(X)$ :

$$
A(\sigma)=\{f(X) / g(X): f, g \in \mathbf{C}[X], g(\alpha) \neq 0 \text { for all } \alpha \in \sigma\} \text {. }
$$

For example, $A(\varnothing)=\mathrm{C}(X), A(\mathrm{C})=\mathrm{C}[X]$. If $\sigma_{1} \subseteq \sigma_{2}$ then $A\left(\sigma_{1}\right) \supseteq A\left(\sigma_{2}\right)$. For all $\sigma$, $\mathrm{C}[X] \subseteq A(\sigma) \subseteq \mathbf{C}(X)$.

Clearly any element $h$ of $A(\sigma)$ determines a rational function on $\mathrm{C}$ : $\alpha \mapsto h(\alpha)$ which has no poles on $\sigma$ and, in fact, $A(\sigma)$ can be identified with the ring of all such rational functions.

If $x$ is an element of an algebra $A$, then any element $h=f / g \in A(\sigma(x))$ $(f, g \in \mathbf{C}[X], g(\alpha) \neq 0$ for $\alpha \in \sigma)$ determines an element $h(x)=f(x) / g(x)$ of $A$ $\left(g(x)\right.$ is invertible in $A$ : indeed let $g(X)=c \prod_{i=1}^{n}\left(X-\lambda_{i}\right), c, \lambda_{i} \in \mathbf{C}$; then for all $i$, $\lambda_{i} \notin \sigma(x)$, i.e., $x-\lambda_{i} \in A^{*}$ and so $\left.g(x) \in A^{*}\right)$.

The following lemma will be used repeatedly without further mention.

LemMA. For $x$ in $A$ and $h \in A(\sigma(x))$ we have $\sigma(h(x))=h(\sigma(x))$.

Proof. Let $h(X)=f(X) / g(X), f, g$ in $\mathrm{C}[X], g(\alpha) \neq 0$ for $\alpha$ in $\sigma$. Then for any scalar $\alpha$ we have

$$
\begin{aligned}
\alpha \in \sigma(h(x)) & \Leftrightarrow \frac{f(x)}{g(x)}-\alpha=\frac{f(x)-\alpha g(x)}{g(x)} \notin A^{*} \Leftrightarrow f(x)-\alpha g(x) \notin A^{*} \\
& \Leftrightarrow \text { The polynomial } f(X)-\alpha g(X) \text { has a root in } \sigma(x) \\
& \Leftrightarrow \exists \beta \in \sigma(x), f(\beta) / g(\beta)=\alpha \Leftrightarrow \alpha \in h(\sigma(x)) .
\end{aligned}
$$

The last lemma shows in particular that for any subset $\sigma$ of $C$ and $f \in A(\sigma)$ we have $\sigma(f)=f(\sigma)$ as $\sigma(X)=\sigma$. 
For an element $x$ in an algebra $A$ we denote by $A(x)$ the following subalgebra of A:

$$
A(x)=\{h(x): h \in A(\sigma(x))\}
$$

Clearly $A(x)$ is the unique subalgebra of $A$ which is minimal with respect to the properties: $x \in A(x), A(x)^{*}=A(x) \cap A^{*}$. Viewing $X$ as an element of the algebra $A(\sigma)$ we have $A(\sigma)=A(\sigma)(X)$.

If $x$ is an element of an algebra $A$ we have a natural homomorphism of algebras

$$
A(\sigma(x)) \rightarrow A(x), \quad f \mapsto f(x) .
$$

This homomorphism is clearly an isomorphism if and only if $x$ is transcendental over $\mathbf{C}$. In case $x$ is algebraic $\sigma(x)$ is the set of roots of the minimal polynomial of $x$ and so it is finite. It follows that $A(x) \cong A(\sigma(x))$ in case $\sigma(x)$ is infinite. In case $\sigma(x)$ is finite there are two possibilities: $x$ is transcendental over $\mathbf{C}$ and then $A(x) \cong A(\sigma(x))$ or $x$ is algebraic and then $A(x) \nRightarrow A(\sigma(x))$.

As we shall see later (Theorem $5(\mathrm{a})(1)), A(x)$ is ordinary if $\sigma(x)$ is finite, so in any case $A(x)$ is ordinary if and only if $A(\sigma(x))$ is ordinary.

The following lemma will serve as our main tool in checking the ordinariness of $A(\sigma)$.

LFMMA 3. Let $x$ be an element of an algebra A. If for every q.m. functional $\varphi$ on $A(x), \varphi\left(x^{2}\right)=(\varphi(x))^{2}$, then $A(x)$ is ordinary.

Proof. Let $\varphi$ be a q.m. functional on $A(x)$. Define

$$
x_{0}= \begin{cases}x & \text { if } \varphi(x) \neq 0 \\ x+1 & \text { if } \varphi(x)=0\end{cases}
$$

Clearly $\psi\left(x_{0}^{2}\right)=\psi\left(x_{0}\right)^{2}$ for any q.m. functional $\psi$.

Set $\varphi\left(x_{0}\right)=\alpha$. Clearly $\alpha \neq 0$. We prove first by induction on $n \geqslant 2$ that $\varphi\left(x_{0}^{n}\right)=\alpha^{n}$. As mentioned, $\varphi\left(x_{0}^{2}\right)=\varphi\left(x_{0}\right)^{2}$, so let us proceed from $n$ to $n+1$. Define a functional $\psi$ on $A(x)$ by $\psi(y)=1 / \alpha^{n-1} \varphi\left(x_{0}^{n-1} y\right)$. Clearly $\psi$ is q.m. and

$$
\psi\left(x_{0}\right)=\varphi\left(x_{0}^{n-1} x_{0}\right) / \alpha^{n-1}=\alpha^{n} / \alpha^{n-1}=\alpha .
$$

Hence

$$
\alpha^{2}=\psi\left(x_{0}^{2}\right)=\varphi\left(x_{0}^{n-1} x_{0}^{2}\right) / \alpha^{n-1}=\varphi\left(x_{0}^{n+1}\right) / \alpha^{n-1},
$$

so $\varphi\left(x_{0}^{n+1}\right)=\alpha^{n+1}$.

It follows that for any polynomial $P(X) \in \mathbf{C}[X]$ we have $\varphi\left(P\left(x_{0}\right)\right)=P(\alpha)$. As $\mathbf{C}\left[x_{0}\right]=\mathbf{C}[x]$ (the subalgebra generated by $x$ ), we see that for any q.m. functional $\psi, \psi \mid \mathbf{C}[x]$ is multiplicative.

Next we prove that if $y \in \mathbf{C}[x]$ is invertible in $A(x)$, then $\varphi\left(y^{-1}\right)=1 / \varphi(y)$. To see this define

$$
\psi(z)=\left(1 / \varphi\left(y^{-1}\right)\right) \varphi\left(y^{-1} z\right)
$$

Clearly $\psi$ is q.m. We have $\psi\left(y^{2}\right)=(\psi(y))^{2}$, so $\left(1 / \varphi\left(y^{-1}\right)\right) \varphi(y)=\left(1 / \varphi\left(y^{-1}\right)\right)^{2}$, that is, $\varphi\left(y^{-1}\right)=1 / \varphi(y)$.

Now, any element of $A(x)$ is a linear combination over $\mathbf{C}$ of an element in $\mathbf{C}[X]$ and elements of the form $1 /(x-\lambda)^{k}$, where $\lambda \in \rho(x), k \geqslant 1$. Hence for $f(X) \in$ $A(\sigma)$ we have $\varphi(f(x))=f(\alpha)$ and $\varphi$ is multiplicative. 
A corollary of Lemma 3 is the fact that ordinariness of $A(\sigma)$ is a monotone property:

Proposition 4. Let $\sigma_{1} \subseteq \sigma_{2} \subseteq$ C. If $A\left(\sigma_{2}\right)$ is ordinary then so is $A\left(\sigma_{1}\right)$.

Proof. Recall $A\left(\sigma_{1}\right) \supseteq A\left(\sigma_{2}\right)$. Let $\varphi$ be a q.m. functional on $A\left(\sigma_{1}\right)$, hence $\varphi \mid A\left(\sigma_{2}\right)$ is a q.m. functional on $A\left(\sigma_{2}\right)$, and since $A\left(\sigma_{2}\right)$ is ordinary, it follows that $\varphi\left(X^{2}\right)=$ $\varphi(X)^{2}$. By Lemma 3, $A\left(\sigma_{1}\right)$ is ordinary.

REMARK. Lemma 3 shows that to check ordinariness of $A(\sigma)$ it is enough to check the restrictions of q.m. functionals on $A(\sigma)$ to $\mathbf{C}[X]$. If $\varphi: \mathbf{C}[X] \rightarrow \mathbf{C}$ is a restriction of a q.m. functional on $A(\sigma)$, then clearly $\varphi(1)=1$ and $\varphi(f) \neq 0$ for any polynomial $f(X)$ in $A(\sigma)^{*}$. Nevertheless, generally a linear functional on $\mathbf{C}[X]$ which fulfils these properties is not extendable to a q.m. functional on $A(\sigma)$. Indeed let $\sigma=\{z \in \mathbf{C}: \operatorname{Re} z \geqslant 0\}$ and let

$$
\varphi: \mathbf{C}[X] \rightarrow \mathbf{C}, \quad 1 \mapsto 1, \quad X \mapsto 1, \quad X^{k} \mapsto 0 \quad(k \geqslant 2) .
$$

Let $\varphi(f)=0, f=\sum_{k=0}^{n} a_{k} X^{k}$, so $a_{0}+a_{1}=0$. We want to show that $f(X)$ has a root in $\sigma$, so we may assume $a_{0} \neq 0$. Let $a_{n} \neq 0, f(X)=a_{n} \Pi_{i=1}^{n}\left(X-\lambda_{i}\right)$. Then as $a_{0}+a_{1}=0$ we have $\prod_{i=1}^{n} \lambda_{i}=\sum_{k=1}^{n}\left(\Pi \lambda_{i} / \lambda_{k}\right)$, or $\sum_{k=1}^{n} 1 / \lambda_{k}=1$. Therefore there exists $k: \operatorname{Re}\left(1 / \lambda_{k}\right)>0$ and so $\operatorname{Re} \lambda_{k}=\operatorname{Re}\left(\bar{\lambda}_{k} / \lambda_{k} \bar{\lambda}_{k}\right)>0, \lambda_{k} \in \sigma . \varphi$ is not extendable to a q.m. functional on $A(\sigma)$ because $A(\sigma)$ is ordinary by Theorem 5(c)(i) below.

TheOREM 5. Let $x$ be an element of an algebra $A, \sigma=\sigma(x), \rho=\rho(x)=\mathrm{C} \backslash \sigma$.

(a) Each of the following conditions implies the ordinariness of $A(x)$.

(1) $|\sigma|<2^{\kappa_{0}}$.

(2) $\sigma$ is a Lebesgue set of measure zero.

(3) $\sigma$ is bounded.

(4) $\sigma$ is not dense in $\mathbf{C}$.

(5) The subfield generated by $\sigma$ is not all $\mathbf{C}$.

(6) For all $a \in \sigma, 0 \neq \alpha \in-(\sigma-a)^{2}, \alpha /(\rho-a) \underline{\sigma} \sigma-a$.

(7) For all $a \in \sigma,-(\sigma-a)^{2} \backslash\{0\} \subseteq(\rho-a) \cdot(\rho-a)$.

(b) If $A(x)$ is ordinary, then:

(1) $|\rho|=2^{\kappa_{0}}$.

(2) The subring generated by $\rho$ is all $\mathbf{C}$.

(3) For all $a \in \sigma$, the additive subgroup generated by $1 /(\rho-a)$ is all $\mathbf{C}$.

(4) The multiplicative subgroup of $\mathbf{C}^{*}$ generated by $(\rho-a) /(\rho-b)$ is all $\mathbf{C}^{*}$ for $a, b$ in $\sigma, a \neq b$.

(c) In each of the following two cases $A(x)$ is ordinary if and only if $\sigma \neq C$ :

(i) $\sigma$ is a closed set.

(ii) $\sigma$ is a subfield of $\mathbf{C}$.

(d) If $\rho$ is a subring of $\mathbf{C}$, then $A(\sigma)$ is ordinary if and only if $\rho=\mathbf{C}$.

(e) If $\sigma$ is an additive subgroup of $\mathrm{C}$, then $A(\sigma)$ is ordinary if and only if it fulfils one of the following equivalent conditions:

(i) $\rho \cdot \rho=\mathbf{C}^{*}$

(ii) For all $\alpha \in \mathbf{C}^{*}, \alpha / \rho \ell \sigma$. 
Proof of Theorem 5. (a)(6) Assume $A(x)$ is not ordinary. Then by Lemma 3 there exists a q.m. functional $\varphi$ on $A(x)$ such that $\varphi\left(x^{2}\right) \neq \varphi(x)^{2}$. Let $a, b \in \sigma$ be such that $\varphi(x)=a, \varphi\left(x^{2}\right)=b^{2}$ and set $\alpha=a^{2}-b^{2}$. Then $0 \neq \alpha=-\varphi\left((x-a)^{2}\right)$ $\in-(\sigma-a)^{2}$.

Let $y=x-a$ and $\lambda \in \mathbf{C}^{*}$. Then

$$
0=\varphi\left(y^{2}-(\lambda+\alpha / \lambda) y+\alpha\right)=\varphi[(y-\lambda)(y-\alpha / \lambda)]
$$

and, since $\varphi$ is q.m., it follows that $\lambda$ or $\alpha / \lambda$ is in $\sigma(y)=\sigma-a$. If $\lambda \in \rho$, then $0 \neq \lambda-a \notin \sigma-a$ and so $\alpha /(\lambda-a) \in \sigma-a$, i.e., $\alpha /(\rho-a) \subseteq \sigma-a$, contradicting the assumption of (a)(6).

$(a)(7)$ is a reformulation of $(a)(6)$.

(a)(1) and (a)(2) follow from (a)(6).

(a)(3). By Lemma 2, $\varphi\left(x^{2}\right)=\varphi(x)^{2}$ for any q.m. functional $\varphi$, so $A(x)$ is ordinary by Lemma 3.

(a)(4) Let $\lambda$ be an interior point of $\rho$. Then $\sigma(1 /(x-\lambda))=1 /(\sigma-\lambda)$ is bounded, so $A(1 /(x-\lambda))=A(x)$ is ordinary by (a)(3).

(a)(5) Assume $A$ is not ordinary and let $F$ be the subfield generated by $\sigma$. By (a)(6) there exist $a \in \sigma$ and $0 \neq \alpha \in-(\sigma-a)^{2} \subseteq F$ such that $\alpha /(\rho-a) \subseteq \sigma-a$ $\subseteq F$. As $0 \neq \alpha \in F$, we have $1 /(\rho-a) \subseteq F, \rho \subseteq F$, contradicting the assumption $F \neq \mathbf{C}$.

In the proof of part (b) we use the fact that $A(x)$ is ordinary if and only if $A(\sigma(x))$ is ordinary, so we assume in each case that the given condition fails to hold and conclude that under this assumption $A(\sigma)$ is not ordinary.

(b)(3) Assume there exists $a \in \sigma$ such that $\langle 1 /(\rho-a)\rangle$ (the additive subgroup generated by $1 /(\rho-a))$ is not all $\mathbf{C}$. Without loss of generality we may assume $a=0$, so $\langle 1 / \rho\rangle \neq \mathbf{C}$. Let $\alpha \in \mathbf{C} \backslash\langle 1 / \rho\rangle$. Clearly $\alpha \neq 0$. Define $\varphi(f(X))=$ $f(0)-f^{\prime}(0) / \alpha$ for $f(X)$ in $A(\sigma)$. Clearly $\varphi$ is a linear functional on $A(\sigma), \varphi(1)=1$. $\varphi$ is not multiplicative because $\varphi(X)=-1 / \alpha \neq 0=\varphi\left(X^{2}\right)$. We claim that $\varphi$ is q.m. Indeed, each invertible element of $A(\sigma)$ is of the form $c f(X) / g(X)$, where $c \in \mathrm{C}^{*}$,

$$
f(X)=\prod_{i=1}^{n}\left(1-\frac{1}{\alpha_{i}} X\right), \quad g(X)=\prod_{j=1}^{m}\left(1-\frac{1}{\beta_{j}} X\right),
$$

with $\alpha_{i}, \beta_{j}$ in $\rho$. Set $t=(f / g)^{\prime}(0)$. Then

$$
\begin{aligned}
t & =\frac{f^{\prime}(0) g(0)-f(0) g^{\prime}(0)}{g^{2}(0)}=f^{\prime}(0)-g^{\prime}(0) \\
& =-\sum_{i=1}^{n} \frac{1}{\alpha_{i}}+\sum_{j=1}^{m} \frac{1}{\beta_{j}} \in\left\langle\frac{1}{\rho}\right\rangle .
\end{aligned}
$$

( $f^{\prime}(0)$ is the coefficient of $X$ in $f(X)$.) Thus $\varphi(f(X) / g(X))=1-t / \alpha \neq 0$ since $\alpha \notin\langle 1 / \rho\rangle$.

It follows that $A(\sigma)$ is not ordinary.

(b)(2) Assume first that the subring generated by $\rho$ is not a field and so it is contained in a valuation ring $R$ for $\mathbf{C}, R \neq \mathbf{C}$ (that is, for any $\alpha$ in $\mathbf{C}^{*}, \alpha \in R$ or $1 / \alpha \in R$; see, e.g., [2, Chapter VI, p. 375]). 
Let $a \in \mathbf{C} \backslash R \subseteq \sigma$. Then for all $\lambda \in \rho \subseteq R, \lambda-a \notin R$ and, hence, $1 /(\lambda-a)$ $\in R$, that is, $1 /(\rho-a) \subseteq R$, and it follows that the additive subgroup generated by $1 /(\rho-a)$ is contained in $R$ and differs from C. By (b)(3) $A(\sigma)$ is not ordinary.

Assume now that the subring generated by $\rho$ is a field $F \neq \mathbf{C}$. Let $\alpha \in \mathbf{C} \backslash F$, $\beta \in \mathbf{C}, \beta^{2}=\alpha$, so $\beta \notin F, \beta^{2} \notin F$. Let $\mathbf{C}=F \oplus V$, where $V$ is an $F$-linear subspace of $\mathbf{C}$ and $\beta \in V$.

We define a $\mathbf{C}$-linear functional $\varphi$ on $A(\sigma)$ by defining it on the linear basis

$$
B=\left\{X^{k}: k \geqslant 0\right\} \cup\left\{1 /(X-\lambda)^{k}: k>1, \lambda \in \rho\right\} .
$$

For $g \in B$ let $\varphi(g)=g(\beta)-2 \pi(g(\beta)$ ) (where $\pi: \mathbf{C} \oplus V \rightarrow V$ is the canonical projection), that is, if $g(\beta)=t+v, t \in F, v \in V$, then $\varphi(g)=t-v$. Clearly $\varphi(1)=1 . \varphi$ is not multiplicative because $\varphi(X)=-\beta$, and if $\beta^{2}=t+v, t \in F$, $v \in V$, then $\varphi\left(X^{2}\right)=t-v \neq t+v=\beta^{2}=\varphi(X)^{2}$ as $\beta^{2} \notin F$.

Any element in $A(\sigma)^{*}$ is of the form $c g$ where $c \in \mathbf{C}^{*}$ and $g \in F(X) \cap A(\sigma)^{*}$ and so $g$ is a linear combination of elements in $B$ with coefficients in $F, \varphi(g)=$ $g(\beta)-2 \pi g(\beta)$. Let $g(\beta)=t+v, t \in F, v \in V$. If $\varphi(g)=t-v=0$, then $t=v$ $=0, g(\beta)=0$, which is impossible as $\beta \in \sigma$. It follows that $\varphi(g) \neq 0$, i.e., $\varphi$ is q.m. and $A(\sigma)$ is not ordinary.

(b)(1) follows from (b)(2).

(b)(4) Let $\alpha \neq 0$ not in the multiplicative subgroup of $\mathbf{C}^{*}$ generated by $(a-\rho) /(b-\rho)$. Clearly $\alpha \neq 1$. Define a functional $\varphi$ on $A(\sigma)$ by $\varphi(f)=$ $(1 /(1-\alpha))(f(a)-\alpha f(b)) \cdot \varphi$ is linear, $\varphi(1)=1$ and $\varphi$ is not multiplicative because $a \neq b$ and multiplicative functionals are linearly independent over $\mathbf{C}$. We claim that $\varphi$ is q.m. Let

$$
f(X)=\frac{\left(X-\lambda_{1}\right) \cdots\left(X-\lambda_{k}\right)}{\left(X-\mu_{1}\right) \cdots\left(X-\mu_{n}\right)}
$$

be in $A(\sigma), \lambda_{i}, \mu_{j} \in \rho$. Then

$$
\begin{aligned}
f(a)-\alpha f(b)= & \frac{\left(a-\lambda_{1}\right) \cdots\left(a-\lambda_{k}\right)}{\left(a-\mu_{1}\right) \cdots\left(a-\mu_{n}\right)}-\alpha \frac{\left(b-\lambda_{1}\right) \cdots\left(b-\lambda_{k}\right)}{\left(b-\mu_{1}\right) \cdots\left(b-\mu_{n}\right)} \\
= & \frac{\left(b-\lambda_{1}\right) \cdots\left(b-\lambda_{k}\right)}{\left(b-\mu_{1}\right) \cdots\left(b-\mu_{n}\right)} \\
& \cdot\left(\frac{\left(a-\lambda_{1}\right) /\left(b-\lambda_{1}\right) \cdots\left(a-\lambda_{k}\right) /\left(b-\lambda_{k}\right)}{\left(a-\mu_{1}\right) /\left(b-\mu_{1}\right) \cdots\left(a-\mu_{n}\right) /\left(b-\mu_{n}\right)}-\alpha\right) \neq 0
\end{aligned}
$$

since $\alpha$ is not in the multiplicative subgroup of $\mathbf{C}^{*}$ generated by $(a-\rho) /(b-\rho)$. It follows that $\varphi$ is q.m. and $A(\sigma)$ is not ordinary.

(c) If $\sigma=\mathrm{C}$, clearly $A(x) \cong \mathrm{C}[X]$ is not ordinary. (i) follows now from (a)(4) and (ii) from (a)(5).

(d) If $\rho=\mathrm{C}$, clearly $A(x) \cong \mathrm{C}(X)$ is ordinary, so (d) follows from (b)(2).

(e)(i) and (ii) are equivalent formulations of the same condition, so we show that (i) is equivalent to ordinariness of $A(\sigma)$. If $A(x)$ is not ordinary then, by (a)(7) $(\rho-a) \cdot(\rho-a) \neq \mathbf{C}^{*}$ for some $a \in \sigma$. As $\sigma$ is an additive group we have $\rho-a=\rho$, so $\rho \cdot \rho \neq \mathbf{C}^{*}$ and (i) does not hold. 
Conversely let $\rho \cdot \rho \neq \mathbf{C}^{*}$, so there exists $\alpha \in \mathbf{C}^{*} \backslash \rho \cdot \rho$. It follows that $\alpha / \rho \subseteq \sigma$, which is an additive subgroup of $\mathbf{C}$. If $\sigma \neq \mathbf{C}$, then $A(x)$ is not ordinary by (b)(3). If $\sigma=\mathrm{C}$, then clearly $A(x)$ is not ordinary.

REMARKS. In the following remarks we show among other things that none of the conditions of Theorem 5(a) (1)-(7) is necessary and none of the conditions of (b) (1)-(4) is sufficient for the ordinariness of $A(x)$.

5(a)(1), (2), (3), (5), (6), (7). For $\sigma=\{z:|z| \geqslant 1\}, A(\sigma)$ is ordinary by 5(c)(i), but $|\sigma|=2^{\aleph_{0}}, \sigma$ is a closed set of infinite measure, $\sigma$ is not bounded, the subfield generated by $\sigma$ is all $\mathbf{C}$ and for any $a \in \sigma,-(\sigma-a)^{2} \backslash\{0\} \mathcal{E}(\rho-a) \cdot(\rho-a)$, so none of the conditions (a)(1), (2), (3), (5), (6), (7) holds in this case.

In 5(a) we may not replace "subfield" by "subring". Indeed let $\boldsymbol{\sigma} \neq \mathbf{C}$ be a valuation ring for $\mathbf{C}$. Then $A(\sigma)$ is not ordinary by (b)(3) as $0 \in \sigma$ and $1 / \rho \subseteq \sigma$.

5(a)(4), (3). Take $\sigma=Q+i Q(Q=$ rationals). Then $A(\sigma)$ is ordinary by (a)(1), but conditions (a)(3) and (a)(4) do not hold.

$5(b)(1),(2)$, (4). We have seen that $A(\sigma)$ is not ordinary in case $\sigma \neq \mathbf{C}$ is a valuation ring for $\mathbf{C}$. In this case conditions (b)(1) and (b)(2) hold as the subring and even the additive subgroup generated by $\rho$ is all $\mathbf{C}$ (if $H \subsetneq G$ are groups, then the subgroup of $G$ generated by $G \backslash H$ is all $G$ ). Also the multiplicative subgroup of $\mathbf{C}^{*}$ generated by $\rho$ is all $\mathbf{C}^{*}$.

We show now that for any $a, b$ in $\sigma, a \neq b$, we have $(\rho-a) /(\rho-b)=C^{*}$, so (b)(4) holds.

For $a \in \sigma, \rho-a=\rho$, so $(\rho-a) /(\rho-b)=\rho / \rho$ for $a, b$ in $\sigma$. Assume $\rho / \rho \neq$ $\mathbf{C}^{*}$ and let $\alpha \in \mathbf{C}^{*} \backslash \rho / \rho$. For any $\lambda \in \rho, \alpha=\lambda \alpha / \lambda$, so $\lambda \alpha \in \sigma$ for all $\lambda \in \rho$, that is, $\rho \subseteq \sigma / \alpha$. Therefore $\mathbf{C}=\sigma \cup \sigma / \alpha, 1 / \alpha \notin \sigma, 1+1 / \alpha \notin \sigma \cup \sigma / \alpha=\mathbf{C}$. This contradiction proves $\rho / \rho=\mathrm{C}^{*}$, so (b)(4) holds.

5(b)(1), (3), (4). Let $\rho \neq \mathbf{C}$ be a subfield of $\mathbf{C},|\rho|=2^{\kappa_{0}}, \sigma=\mathbf{C} \backslash \rho$. Then $A(\sigma)$ is not ordinary by (b)(2), but (b)(1) holds and for any $a \in \sigma$ the additive subgroup generated by $1 /(\rho-a)=\sigma$ is all $C$, so (b)(3) also holds. (b)(4) holds by the argument in the preceding remark.

In 5(b)(3) we may not replace "additive subgroup" by "additive subsemigroup". Indeed for $\sigma=\{z \in \mathrm{C}: \operatorname{Re} z \leqslant 0\}, A(\sigma)$ is ordinary by 5(c)(i). Nevertheless for all $a \in \sigma$ the subsemigroup generated by $1 /(\rho-a)$ is not all $\mathbf{C}$ since $1 /(\rho-a) \subseteq \rho$ and $\rho \neq \mathbf{C}$ is a semigroup.

In $5(\mathrm{~b})(2)$ we may not replace "subring" by "additive subgroup". Indeed by the next claim, for $\rho=\mathbf{R}+i Q, A(\sigma)$ is ordinary.

Claim. Let $V$ be a dense subset of the real field $\mathbf{R}, \rho=\mathbf{R}+i V, \boldsymbol{\sigma}=\mathbf{C} \backslash \rho$. Then $A(\sigma)$ is ordinary.

Proof. We shall show that for all $\alpha \in \mathbf{C},(\rho-\alpha) \cdot(\rho-\alpha) \supseteq \mathbf{C}^{*}$ and the claim will follow from Theorem 5(a)(7). For any $\alpha \in \mathbf{C}, \mathbf{R}+i V-\alpha$ is of the form $\mathbf{R}+i V_{1}$, where $V_{1}$ is a dense subset of $\mathbf{R}$, so it suffices to show that

$$
(\mathbf{R}+i V) \cdot(\mathbf{R}+i V) \supseteq \mathbf{C}^{*}
$$

for a dense subset $V$ of $\mathbf{R}$. Let $a+i b \in \mathbf{C}^{*}, a, b \in \mathbf{R}$. We have to find $x, y$ in $\mathbf{R}$ and $v, w$ in $V$ such that $a+i b=(x+i v)(y+i w)$, that is,

$$
x y-v w=a, \quad w x+v y=b .
$$


There exist elements $v$ and $w \neq 0$ in $V$ such that $b^{2}-4 v w(a+v w)>0$ because $V$ is dense in $\mathbf{R}$. Now we can solve the above system of equations for $x$ and $y$ : $w x^{2}+v x y=b x, w x^{2}-b x+v(a+v w)=0$. There exists a real solution $x \neq 0$ to the last equation as its discriminant $b^{2}-4 v w(a+v w)$ is positive and $w \neq 0$. We also find $y=(a+v w) / x$.

The last claim provides us with an example of an additive subgroup of $\mathbf{C}$, which is an $F_{\sigma}$ set of measure zero, $\rho=\mathbf{R}+i Q$ such that $A(\sigma)$ is ordinary (cf. also Theorem $5(\mathrm{a})(2)$ and $5(\mathrm{~b})(3))$. Therefore the Lebesgue measure cannot be used to characterize ordinariness of $A(\sigma)$ for $\sigma$ Lebesgue measurable.

The following three examples again illustrate this point.

Example of a subset $\sigma$ of $\mathbf{C}$ such that $A(\sigma)$ is ordinary, but $A(\sigma \cup\{0\})$ is not ordinary.

Set $\rho=\{1 / z: 0 \neq z \in \mathbf{R}+i Q\} \cup\{0\}$ and $\sigma=\mathbf{C} \backslash \rho . X \in A(\sigma)^{*}$ and $A(\sigma)=$ $A(\sigma)(1 / X), \sigma(1 / X)=\mathbf{C} \backslash(\mathbf{R}+i Q)$, so $A(\sigma)$ is ordinary.

Set $\sigma^{\prime}=\sigma \cup\{0\}$. Then $\rho^{\prime}=\mathbf{C}-\sigma^{\prime}=\{1 / z: 0 \neq z \in \mathbf{R}+i Q\}, 0 \in \sigma^{\prime}$ and $1 / \rho^{\prime} \subseteq \mathbf{R}+i Q$, which is an additive subgroup of $\mathbf{C}$. By Theorem $5(\mathrm{~b})(3) A\left(\sigma^{\prime}\right)$ is not ordinary.

ExAmple. Let $\mathbf{C}=Q \oplus V$, where $V$ is a $Q$-linear subspace of $\mathbf{C}$. Set $\rho=$ $\{1 / v: 0 \neq v \in V\}, \sigma=\mathbf{C} \backslash \rho$. By Theorem 5(b)(3), $A(\sigma)$ is not ordinary, although the outer measure of $\rho$ is infinite.

ExAmple. If $\sigma \neq \mathrm{C}$ is a valuation ring for $\mathrm{C}$, then $A(\sigma)$ is not ordinary, but the inner measure of $\sigma$ is 0 (cf. Theorem $5(\mathrm{a})(2)$ ).

In spite of the preceding examples it is not clear whether $A(\sigma)$ is ordinary for $\rho=\mathbf{C} \backslash \sigma$ of positive inner measure.

Let $\mathcal{S}=\{\sigma \subseteq \mathrm{C}: A(\sigma)$ is ordinary $\}$. By Theorem 5(a) we know that $\mathcal{S}$ contains all the nondense subsets of $\mathbf{C}$, all the subfields, etc. By the monotone property (Proposition 4) if $\sigma_{1} \subseteq \sigma_{2} \in \mathcal{S}$, then $\sigma_{1}$ is also in $\mathcal{S}$.

For any $\boldsymbol{\sigma} \subseteq \mathbf{C}$ any Möbius transformation of $\mathbf{C}$ of the form

$$
T z=(a z-b) /(c z-d) \text { where } a d-b c \neq 0
$$

and $d / c \notin \sigma$ if $c \neq 0$, induces an isomorphism $A(\sigma) \cong A(T(\sigma))$. (In fact any isomorphism $A(\sigma) \cong a\left(\sigma^{\prime}\right)$ is induced by a Möbius transformation $T$ as above with $\sigma^{\prime}=T(\sigma)$.) It follows that if $\sigma \in \mathcal{S}$, then $T \sigma$ is also in $\mathcal{S}$ for $T$ as above.

If $\theta$ is an automorphism of $\mathbf{C}$ it is easy to show for $\sigma \subseteq \mathbf{C}$ that $A(\sigma)$ is ordinary if and only if $A(\theta(\sigma))$ is ordinary, although generally $A(\sigma) \cong A(\theta(\sigma))$. Therefore if $\sigma \in \mathcal{S}$, then $\theta(\sigma) \in \mathcal{S}$.

On the other hand $\mathcal{S}$ is not closed under finite unions: for $\sigma_{1}=\{z:|z| \leqslant 1\}$ and $\sigma_{2}=\mathrm{C} \backslash \sigma_{1}, \sigma_{1}, \sigma_{2}$ are in $\mathcal{S}$ but $\sigma_{1} \cup \sigma_{2}=\mathrm{C} \notin \mathcal{S}$. As we have seen above, it is possible for $\sigma \subseteq \mathbf{C}$ and $c \in \mathbf{C}: \sigma \in \mathcal{S}$, but $\sigma \cup\{c\} \notin \mathcal{S}$.

4. The algebra of entire functions. The algebra $E$ of entire analytic functions drove our attention because of its simple spectral structure: by the well-known theorem of Picard, for each $f \in E \backslash \mathbf{C}, \rho(f)$ is either empty or consists of a single point. Other equivalent well-known formulations of the Picard theorem are the 
following: if $f$ and $g$ are nonconstant entire functions then $e^{f}+e^{g} \neq 1$; or, if $g_{1}$, $g_{2}, g_{3}$ are three different entire functions with $g_{i}(0)=0$ for $i=1,2,3$, then the functions $e^{g_{1}}, e^{g_{2}}, e^{g_{3}}$ are linearly independent.

Actually a much stronger result holds: in his proof of the Picard theorem, Borel proved the following theorem (see [1, p. 387] and also [5, p. 118]):

THEOREM (BOREL). The set $\left\{e^{g}: g\right.$ entire with $\left.g(0)=0\right\}$ is linearly independent.

In other words a set of invertible entire functions is linearly independent, provided any two different elements in the set are linearly independent.

We present first the proof of the equivalence of the formulations of the Picard theorem in the general case.

Proposition 6. The following two properties are equivalent for an algebra $A$ :

(i) For all $x \in A \backslash \mathbf{C}, \rho(x)$ consists of at most one point.

(ii) Each set of three different elements of $A^{*}$ is linearly independent, provided each pair of its elements is linearly independent.

Proof. (i) $\Rightarrow$ (ii) Let $x, y, z$ be three elements as in (ii) and assume $a x+b y+c z$ $=0, a, b, c$ not all zero. Therefore $a \neq 0, b \neq 0, c \neq 0$. We have $-a x z^{-1}=$ $b y z^{-1}+c$, so 0 and $-c$ are in $\rho\left(b y z^{-1}\right)$, but byz $z^{-1} \notin \mathbf{C}$ as $y$ and $z$ are linearly independent. This contradicts (i).

(ii) $\Rightarrow$ (i) Let $\alpha \neq \beta$ be in $\rho(x), x \in A \backslash$ C. Then $1, x-\alpha, x-\beta$ are in $A^{*}$, they are mutually independent, but not independent.

There is a natural topology on the algebra $E$ of entire functions-the topology of uniform convergence on compact sets. It turns out that although $E$ is not ordinary, q.m. functionals which are continuous with respect to this topology are multiplicative. In the proof of part 2 of the following theorem we use the ideas from the proof of Theorem 10.9 in [6].

THEOREM 7.' Let $E$ be the algebra of entire functions.

1. Each multiplicative functional $\varphi$ on $E$ is of the form $\varphi(f)=f(a)$ for a unique $a \in \mathbf{C}$.

2. A q.m. continuous functional on $E$ is multiplicative.

3. $E$ is not ordinary. Moreover, the cardinality of the set of q.m. functionals on $E$ is $2^{2^{\mu_{0}}}$ while the cardinality of the set of multiplicative functionals on $E$ is $2^{\kappa_{0}}$.

Proof. 1. Let $\varphi$ be a multiplicative functional on $E$. Set $a=\varphi(z)$ ( $z$ is the function $f(z)=z)$. Let $f \in E$. Then $f(z)-f(a)=(z-a) g(z)$ for some $g \in E$. Hence

$$
\varphi(f-f(a))=\varphi((z-a) g)=\varphi(z-a) \varphi(g)=(\varphi(z)-a) \varphi(g)=0,
$$

that is $\varphi(f)=f(a)$.

$a$ is uniquely determined by $\varphi: a=\varphi(z)$.

\footnotetext{
1 ADDED IN PROOF. Professor W. Żelazko (Warsaw) informed us that the results of this theorem have appeared already in his paper: 7.2 in [7] and 7.3 in Colloq. Math. 28 (1973), 251-253.
} 
2. We need the following two lemmas:

LEMMA a. Let $g$ be an entire function, $g(0)=1, g^{\prime}(0)=0$ and $0<|g(z)| \leqslant e^{\alpha|z|}$ for all $z \in \mathbf{C}$ and some $\alpha>0$. Then $g(z)=1$ for all $z \in \mathbf{C}$.

Proof. Set $f(z)=g(z / \alpha)$ in Lemma 10.8 of [6].

LEMMA b. If $\left(\alpha_{n}\right)_{n=0}^{\infty} \subseteq \mathbf{C}$ is a sequence such that for each sequence $\left(a_{n}\right)_{n=0}^{\infty} \subseteq \mathbf{C}$ with $\lim _{n \rightarrow \infty}\left|a_{n}\right|^{1 / n}=0, \lim _{n \rightarrow \infty} a_{n} \alpha_{n}=0$ holds, then there exists $\alpha \in \mathbf{R}^{+}$, such that $\left|\alpha_{n}\right| \leqslant \alpha^{n}$ for all $n$.

Proof. Assume there exists no such $\alpha$. Then there exists a subsequence $\left(\alpha_{n_{k}}\right)$ of $\left(\alpha_{n}\right)$ such that $\left|\alpha_{n_{k}}\right| \geqslant k^{n_{k}}$ for all $k \geqslant 1$. Define $a_{n_{k}}=1 / \alpha_{n_{k}}, a_{n}=0$ for $n \notin\left\{n_{k}\right\}$. Clearly $\lim _{n \rightarrow \infty} a_{n}^{1 / n}=0$, but $a_{n_{k}} \alpha_{n_{k}}=1$ for all $k$. This contradiction proves the lemma.

Proof of Theorem 7.2. Let $\varphi$ be a continuous q.m. functional on $A$. We may assume $\varphi(z)=0$, since otherwise we consider $\psi(f)=\varphi(f(z-\varphi(z)))$. Set $\varphi\left(z^{n}\right)=$ $\alpha_{n}, n=0,1,2, \ldots$

If $\left(a_{n}\right) \subseteq \mathbf{C}$ is a sequence such that $\lim _{n \rightarrow \infty}\left|a_{n}\right|^{1 / n}=0$, then $f(z)=\sum_{n-0}^{\infty} a_{n} z^{n}$ is in $E$, and by the continuity of $\varphi, \varphi(f)=\sum_{n=0}^{\infty} a_{n} \alpha_{n}$, so by Lemma $b,\left|\alpha_{n}\right|<\alpha^{n}$ $(n=0,1, \ldots)$ for some positive $\alpha$.

Set

$$
g(z)=\sum_{n=0}^{\infty} \frac{\alpha_{n}}{n !} z^{n}
$$

Then

$$
\left|\frac{\alpha_{n}}{n !}\right|^{1 / n} \leqslant \frac{\alpha}{(n !)^{1 / n}} \underset{n \rightarrow \infty}{\rightarrow} 0,
$$

that is, $g \in E$. We have $g(0)=\alpha_{0}=1, g^{\prime}(0)=\alpha_{1}=0$ and also

$$
|g(z)| \leqslant \sum_{n=0}^{\infty} \frac{\left|\alpha_{n}\right|}{n !}|z|^{n} \leqslant \sum_{n=0}^{\infty} \frac{\alpha^{n}|z|^{n}}{n !}=e^{\alpha|z|}
$$

Also, for all $\lambda \in \mathbf{C}$,

$$
\varphi\left(e^{\lambda z}\right)=\varphi\left(\sum_{n=0}^{\infty} \frac{\lambda^{n} z^{n}}{n !}\right)=\sum_{n=0}^{\infty} \frac{\lambda^{n} \alpha_{n}}{n !}=g(\lambda),
$$

and since $e^{\lambda z} \in E^{*}$ and $\varphi$ is q.m. it follows that $g(\lambda) \neq 0$ for all $\lambda \in \mathbf{C}$. By Lemma a, $g(z)=1$ for all $z \in \mathrm{C}$, that is, $\alpha_{n}=0$ for all $n \geqslant 1$ and so, as $\varphi$ is continuous, $\varphi(f)=f(0)$ for all $f \in E$.

Note that from the proof it follows that a continuous linear functional on $E$ which does not vanish on elements of the form $e^{\lambda z}, \lambda \in \mathbf{C}$ is multiplicative.

3. By the above-mentioned theorem of Borel one can define a linear functional on $E$ by assigning arbitrary nonzero values to the elements of the set $\left\{e^{g}: g \in E\right.$, $g(0)=0\}$ and then extending it to the whole of $E$. Such functionals are q.m. since each element of $E^{*}$ is of the form $c e^{g}, c \in \mathbf{C}^{*}, g(0)=0$. Hence there exist $2^{2^{n_{0}}}$ q.m. functionals, and by 1 , just $2^{N_{0}}$ multiplicative ones. 
Example 8. We present now an example of an algebra $A$ such that for each $x \in A \backslash \mathbf{C}, \rho(x)$ consists of at most one point (that is, $A$ fulfils the analogue of Picard's theorem), but in contrast to $E, A$ is ordinary (so, in particular, $A$ does not fulfil the analogue of Borel's theorem).

Let $A$ denote the algebra of all elements in the ring of rational functions $\mathbf{C}(X, Y)$ of the form $f(X, Y) / \mathrm{II}_{i=1}^{n}\left(X+\lambda_{i} Y+\lambda_{i}^{2}\right)$, where $f(X, Y) \in \mathbf{C}[X, Y]$ (the ring of polynomials in $X, Y)$ and $\lambda_{i} \in \mathbf{C}$. Let $\varphi$ be a linear functional on $A$ with $\varphi(1)=1$. Then $\varphi\left(X+\lambda Y+\lambda^{2}\right)=\varphi(X)+\lambda \varphi(Y)+\lambda^{2}=0$ for a suitable $\lambda$ in C. Since $X+\lambda Y+\lambda^{2} \in A^{*}$, it follows that there are no q.m. functionals on $A$, so $A$ is ordinary vacuously.

Assume that the property $|\rho(x)| \leqslant 1$ for all $x$ in $A$ does not hold. Then by Proposition 6 there exist three elements $f, g, h$ in $A^{*}$, any two of which being linearly independent such that $a f+b g+c h=0, a, b, c$ in $\mathbf{C}$ not all zero so $a \neq 0$, $b \neq 0, c \neq 0$. By multiplying $f, g, h$ with their common denominator we may assume $f, g, h$ are polynomials in $\mathbf{C}[X, Y]$. Furthermore we may assume that no two of the polynomials $f, g, h$ have prime factors in common in $\mathbf{C}[X, Y]$ and that they are of the form

$$
\begin{gathered}
f(X, Y)=\prod_{i=1}^{n}\left(X+\lambda_{i} Y+\lambda_{i}^{2}\right), \quad g(X, Y)=\prod_{i=1}^{m}\left(X+\mu_{i} Y+\mu_{i}^{2}\right), \\
h(X, Y)=\prod_{i=1}^{k}\left(X+\nu_{i} Y+\nu_{i}^{2}\right) .
\end{gathered}
$$

We may assume $g \notin \mathbf{C}, f \notin \mathbf{C}$, so $n \geqslant 1, f\left(-\lambda_{1} Y-\lambda_{1}^{2}, Y\right)=0$,

$$
\begin{gathered}
b g\left(-\lambda_{1} Y-\lambda_{1}^{2}, Y\right)+\operatorname{ch}\left(-\lambda_{1} Y-\lambda_{1}^{2}, Y\right)=0 . \\
g\left(-\lambda_{1} Y-\lambda_{1}^{2}, Y\right)=\prod_{i=1}^{m}\left(\left(\mu_{i}-\lambda_{1}\right) Y+\mu_{i}^{2}-\lambda_{1}^{2}\right),
\end{gathered}
$$

so $-\left(\mu_{1}^{2}-\lambda_{1}^{2}\right) /\left(\mu_{1}-\lambda_{1}\right)=-\left(\mu_{1}+\lambda_{1}\right)$ is a root of $g\left(-\lambda_{1} Y-\lambda_{1}^{2}, Y\right)$ as a polynomial in $\mathbf{C}[Y]$. It follows that $-\left(\mu_{1}+\lambda_{1}\right)$ is also a root of $h\left(-\lambda_{1} Y-\lambda_{1}^{2}, Y\right)$ so there exist $1 \leqslant i \leqslant k:-\left(\mu_{1}+\lambda_{1}\right)=-\left(\nu_{i}+\lambda_{1}\right), \mu_{1}=\nu_{i}$ contradicting the fact that $g$ and $h$ have no prime factors in common in $\mathbf{C}[X, Y]$.

It is easy to show that the algebra

$$
B=\left\{\frac{f(X, Y, Z)}{X^{k} Y^{m}(X+Y+Z)^{l}}: f(X, Y, Z) \in \mathbf{C}[X, Y, Z]\right\} \subseteq \mathbf{C}(X, Y, Z)
$$

fulfils the Picard property, does not fulfil the Borel property and is not ordinary. We conclude that for general algebras the only connections between the three properties we have studied here are the following: the Borel property implies the Picard property and nonordinariness. On the other hand for algebras of the type $A(x)$ it is easy to show that the Picard property and the Borel property are equivalent.

For comparison with the Picard property we include here the following

Proposition 9. Let $A \neq \mathbf{C}$ be an algebra such that $\rho(x)=\varnothing$ for all $x \in A \backslash \mathbf{C}$ (that is, there are no invertible elements apart from nonzero scalars). Then $A$ is not ordinary. 
Proof. Let $A=\mathrm{C} \oplus V$, where $V$ is a linear subspace of $A$. Let $0 \neq v_{0} \in V$. If $v_{0}^{2} \notin \mathbf{C}+\mathbf{C} v_{0}$, then clearly we may define a linear functional such that $\varphi(1)=1$, $\varphi\left(v_{0}^{2}\right) \neq \varphi\left(v_{0}\right)^{2}$, and so $A$ is not ordinary. Assume $v_{0}^{2}=\alpha+\beta v_{0}$. Choose a scalar $c_{0}$ such that $c_{0}^{2} \neq \alpha+\beta c_{0}$ and let $\varphi$ be a linear functional such that $\varphi(1)=1$, $\varphi\left(v_{0}\right)=c_{0}$ and so $\varphi\left(v_{0}^{2}\right) \neq \varphi\left(v_{0}\right)^{2} . A$ is not ordinary.

On the other hand if there exists in an algebra $A$ an element $x$ such that $\sigma(x)=\varnothing$, then $A$ is ordinary vacuously (there are no q.m. functionals on $A$ ). The existence of $x$ with $\sigma(x)=\varnothing$ is equivalent to the property: $A$ contains a field $F \supsetneq \mathbf{C}$.

5. Remarks on the global case. Given an algebra $A$, which conditions on $A$ imply ordinariness? Clearly such a condition is " $A(x)$ is ordinary for all $x$ in $A$ " and so we can globalize Theorem 5. For example if $\sigma(x)$ is of measure zero or nondense in $C$ for any $x$ in $A$, then $A$ is ordinary. Nevertheless $A$ may be ordinary and $A(x)$ not ordinary for any $x \in A \backslash \mathrm{C}$ (see Example 8 above). This shows that the class of ordinary algebras is not closed with respect to subalgebras, although, as it is easy to show, it is closed with respect to homomorphic images. Even in ordinary algebras of the type $A(\sigma)$ may exist elements $x$ such that $\rho(x)=\varnothing$ and so $A(x) \cong \mathrm{C} X]$ is not ordinary. Furthermore we have the following

Example of an ordinary algebra $A(\sigma)$ such that any element $x$ in $A(\sigma)$ is a sum $x=y+z$, with $\rho(y)=\rho(z)=\varnothing$.

Let $\sigma=\{z \in \mathrm{C}:|z| \geqslant 1\}$. Then $A(\sigma)$ is ordinary by Theorem 5(c)(i). Let $f / g \in A(\sigma)$, where $f, g$ are in $\mathrm{C}[X], g \in A(\sigma)^{*}$. Let $n=\max (\operatorname{deg} f, \operatorname{deg} g$ ) (take $n=\operatorname{deg} g$ for $f=0$ ). We have $f / g=y+z$ where

$$
y=\frac{f+X^{n+2}+(n+2) X^{n+1}}{g} \text { and } z=-\frac{X^{n+2}+(n+2) X^{n+1}}{g} \text {. }
$$

If $\beta_{i}(1 \leqslant i \leqslant n+2)$ are the roots of the polynomial $X^{n+2}+(n+2) X^{n+1}+f(X)$ $-\alpha g(X)$, then $\sum_{i=1}^{n+2} \beta_{i}=-(n+2)$, so there exists $i:\left|\beta_{i}\right|>1, \beta_{i} \in \sigma$. It follows that $\alpha=y\left(\beta_{i}\right) \in y(\sigma)=\sigma(y)$, so $\sigma(y)=\mathbf{C}$. Similarly $\sigma(z)=\mathbf{C}$.

The algebra $A=\mathrm{C}(X)[Y]$ is also ordinary and any element $x$ in $A$ is a sum $x=y+z$, where $\rho(y)=\rho(z)=\varnothing$.

Proposition 10. The following conditions on an algebra $A$ are equivalent:

(i) The spectrum of every element in $A$ is bounded.

(ii) The spectrum of every element in $A$ is closed and differs from $\mathbf{C}$.

(iii) The spectrum of every element in $A$ is compact.

Under these conditions $A$ is ordinary.

Proof. (i) $\Rightarrow$ (ii) Assume there is $x$ in $A$ such that $\sigma(x)$ is not closed and let $\alpha \in \overline{\sigma(x)} \backslash \sigma(x)$. Then $x-\alpha \in A^{*}$ and $\sigma(1 /(x-\alpha))=1 /(\sigma-\alpha)$ is not bounded, contradicting (i).

(ii) $\Rightarrow$ (i) Assume there is $x$ in $A$ such that $\sigma(x)$ is not bounded. Let $\alpha \in \mathbf{C} \backslash \sigma(x)$ $=\rho(x)$. Then $\sigma(1 /(x-\alpha))=1 /(\sigma-\alpha)$ is not closed because

$$
0 \in \overline{\sigma(1 /(x-\alpha))} \backslash \sigma(1 /(x-\alpha)) .
$$

ExAMPLE. For $\sigma=\mathbf{R}$ it can be shown that the spectrum of any element in $A(\sigma)$ is not dense in $\mathbf{C}$, but $\sigma(X)=\mathbf{R}$ is not bounded. 
If we assume $\sigma(x) \neq \mathbf{C}$ for all $x$ in $A$ (that is $\rho(x) \neq \varnothing$ ), but drop the assumption that $\sigma(x)$ is closed in Proposition 10(ii), then the next example shows that $A$ may be not ordinary (even if $A$ is of the type $A(\sigma)$ ). This holds in spite of the fact that in this case $\rho(x)$ is a "large set"-for all $x$ in $A, \rho(x)+\rho(x)=C$. (Indeed let $x \in A$ and $a \in \mathbf{C}$. If $\lambda \in \rho\left(x^{2}-a x\right)$, then $x^{2}-a x-\lambda=(x-\alpha)(x-\beta) \in A^{*}$, hence $\alpha, \beta \in \rho(x)$ and $\alpha+\beta=a$, that is, $\mathbf{C}=\rho(x)+\rho(x)$.)

EXAMPLE. Let $R \neq \mathbf{C}$ be an integrally closed domain whose field of quotients is C. (E.g. take for $R \neq \mathbf{C}$ a valuation ring for $\mathbf{C}$. We recall that an integral domain $R$ is called integrally closed if any element of the field of quotients of $R$ which is a root of a monic polynomial over $R$ belongs to $R$.)

Let $\rho=\{1 / r: 0 \neq r \in R\}$ and $\sigma \in \mathbf{C} \backslash \rho$. Then $0 \in \sigma, 1 / \rho \subseteq R \varsubsetneqq \mathrm{C}$, so $A(\sigma)$ is not ordinary by Theorem $5(b)(3)$.

Claim. A polynomial $f(X)$ is in $A(\sigma)^{*}$ if and only if $f(X)=c_{0} g(X)$ for $c_{0} \in \mathbf{C}^{*}$ and $g(X) \in R[X]$ with $g(0)=1$.

Proof. Let $f(X) \in A(\sigma)^{*}$,

$$
f(X)=c \prod_{i=1}^{n}\left(X-\frac{1}{r_{i}}\right), \quad 0 \neq r_{i} \in R .
$$

Set

$$
c_{0}=\frac{c(-1)^{n}}{\prod_{i=1}^{n} r_{i}}, \quad g(X)=(-1)^{n} \prod_{i=1}^{n}\left(r_{i} X-1\right) .
$$

Then $f(X)=c_{0} g(X), g(X) \in R[X], g(0)=1$. Conversely, let $g(X) \in R[X], g(0)$ $=1$, that is, $g(X)=\sum_{k=0}^{n} r_{k} X^{k}, r_{k} \in R, r_{0}=1$. Let $\lambda \in \mathbf{C}$ be a root of $g(X)$. Then $X^{n} g(1 / X)=\sum_{k=0}^{n} r_{k} X^{n-k}$ is a monic polynomial in $R[X]$ and $1 / \lambda$ is a root of this polynomial, as $\left(1 / \lambda^{n}\right) g(1 /(1 / \lambda))=\left(1 / \lambda^{n}\right) g(\lambda)=0$. As $R$ is integrally closed, we have $1 / \lambda \in R, \lambda \in \rho$, so $g(X) \in A(\sigma)^{*}$. This completes the proof of our claim.

Let $0 \neq x$ be any element of $A$. By the claim above and using the fact that $\mathbf{C}$ is the field of quotients of $R$, we see that $x$ is of the form $x=c f / g$, where $c \in C^{*}$, $f(\dot{X}), g(X)$ are in $R[X], g(0)=1$.

Then for $\lambda=c(f(0)-1)$ we have $x-\lambda=c(f(X)-g(X)(f(0)-1)) / g(X)$. By the claim above the numerator is in $A(\sigma)^{*}$, so $\lambda \in \rho(x), \rho(x) \neq \varnothing$.

We conclude from the last two sections that the spectral approach is not satisfactory in the global case.

\section{REFERENCES}

1. E. Borel, Sur les zéros des fonctions entières, Acta. Math. 20 (1896), 357-396.

2. N. Bourbaki, Commutative algebra, Addison-Wesley, Reading, Mass., 1972.

3. A. M. Gleason, A characterization of maximal ideals, J. Analyse Math. 19 (1967), 171-172.

4. J. P. Kahane and W. Zelazko, A characterization of maximal ideals in commutative Banach algebras, Studia Math. 29 (1968), 339-343.

5. R. Nevanlinna, Le théorème de Picard-Borel, Chelsea, New York, 1974.

6. W. Rudin, Functional analysis, McGraw-Hill, New York, 1973.

7. W. Zelazko, A characterization of multiplicative linear functionals in complex Banach algebras, Studia Math. 30 (1968), 83-85.

Department of Mathematics, University of Haifa, Mt. Carmel, Haifa, Israel 